\title{
miR-181a modulates proliferation, migration and autophagy in AGS gastric cancer cells and downregulates MTMR3
}

 \\ ${ }^{1}$ Department of Gastroenterology, Guangzhou Digestive Disease Center, Guangzhou Key Laboratory of Digestive Disease, \\ Guangzhou First People's Hospital, Guangzhou Medical University, Guangzhou, Guangdong 510180; \\ ${ }^{2}$ School of Public Health, Guangzhou Medical University, Guangzhou, Guangdong 511436; ${ }^{3}$ Department of \\ General Surgery, Guangzhou Digestive Disease Center, Guangzhou First People's Hospital, \\ Guangzhou Medical University, Guangzhou, Guangdong 510180, P.R. China
}

Received December 6, 2015; Accepted January 2, 2017

DOI: $10.3892 / \mathrm{mmr} .2017 .6289$

\begin{abstract}
MicroRNAs (miRs) have emerged as crucial regulators of tumorigenesis by regulating post-transcriptional gene expression. miR-181a was previously demonstrated to be overexpressed in human gastric cancer tissues and cell lines, whereas MTMR3 was underexpressed. The MTMR3 gene was identified as a direct target of miR-181a. However, its functional role in gastric cancer remains to be established. In the present study, miR-181a was demonstrated to inhibit MTMR3 expression in AGS cells. Ectopic expression of miR-181a mimics or introduction of MTMR3 small interfering RNA resulted in an increase in cell proliferation, colony formation, migration, invasion, as well as suppression of apoptosis. Further investigation in the present study indicated that overexpression of miR-181a, or depletion of MTMR3, attenuated starvation-induced autophagy in AGS cells. In addition, inhibition of endogenous miR-181a led to stimulation of autophagic activity. Collectively, these data suggest that miR-181a is a novel regulator of gastric cancer progression and autophagy, and miR-181a modulation may be a potential strategy for the development of miRNA-based therapeutics for gastric cancer.
\end{abstract}

\section{Introduction}

Gastric cancer (GC) is the fourth most common malignancy and the third most common cause of cancer-associated mortality worldwide (1). The development of GC is a complex

Correspondence to: Professor Yuqiang Nie, Department of Gastroenterology, Guangzhou Digestive Disease Center, Guangzhou Key Laboratory of Digestive Disease, Guangzhou First People's Hospital, Guangzhou Medical University, 1 Panfu Road, Guangzhou, Guangdong 510180, P.R. China

E-mail: nie_yuqiang@21cn.com

Key words: miR-181a, gastric cancer, myotubularin related protein 3 , autophagy, carcinogenesis and multistep process. It results from a combination of environmental factors and accumulation of generalized and specific genetic alterations (2). MicroRNAs (miRNAs or miRs) serve key roles in post-transcriptional regulation predominantly by pairing to the 3'-untranslated regions (UTRs) of target mRNA (3). Accumulating evidence suggests that aberrantly-expressed miRNAs are involved in the initiation, progression and metastasis of a variety of cancers, including GC (4-6). Therefore, identification of genes that regulate tumorigenesis and GC progression are currently under intense investigation.

The present study investigated miRNAs using microarray and reverse transcription-quantitative polymerase chain reaction (RT-qPCR) analyses. Previous work of the authors has confirmed that miRNA-181a (miR-181a) was significantly upregulated, whereas myotubularin related protein 3 (MTMR3) was notably downregulated in human GC tissues and cell lines (7). In addition, ectopic overexpression of miR-181a promoted tumor progression in SGC-7901 cells (8). Furthermore, MTMR3 was identified as a direct target of miR-181a. The MTMR3 gene is an essential component of autophagy (9). However, to date, the role of MTMR3 in GC remains unclear.

In the current study, aimed to determine the effect of miR-181a on MTMR3 expression in AGS cells. In addition, the effect of miR-181a overexpression or MTMR3 knockdown on proliferation, colony formation, migration and invasion, and apoptosis of AGS cells was assessed. Furthermore, the effect of miR-181a and MTMR3 in autophagy was investigated. The results presented provide evidence that miR-181a and MTMR3 serve important roles in GC development.

\section{Materials and methods}

Cell culture. The AGS human gastric cancer cell line was purchased from the Cell Bank of Type Culture Collection of Chinese Academy of Sciences (Shanghai, China). Cells were cultured in RPMI-1640 medium supplemented with $10 \%$ fetal bovine serum, $50 \mathrm{U} / \mathrm{ml}$ penicillin and $50 \mu \mathrm{g} / \mathrm{ml}$ streptomycin (Gibco; Thermo Fisher Scientific, Inc., Waltham, MA, USA) at $37^{\circ} \mathrm{C}$ in humidified $5 \% \mathrm{CO}_{2}$ atmosphere. 
$R N A$ extraction and $R T-q P C R$. Total RNA was extracted from AGS cells using TRIzol reagent (Invitrogen; Thermo Fisher Scientific, Inc.). Reverse transcription was conducted using the GoScript Reverse Transcription system (Promega Corporation, Madison, WI, USA). RT-qPCR was performed using SYBR-Green qPCR SuperMix (Invitrogen; Thermo Fisher Scientific, Inc.). 18S rRNA was used as an internal control. PCR primers used were as follows: MTMR3, forward 5'-GCAGGACCAGATATGTGAGAGA-3' and reverse 5'-GCA CACAACATGCAGATGAG-3'; 18S rRNA, forward 5'-CCT GGATACCGCAGCTAGGA-3' and reverse 5'-GCGGCGCAA TACGAATGCCCC-3'. RT-qPCR was performed at $95^{\circ} \mathrm{C}$ for $10 \mathrm{~min}$, followed by 40 cycles of $95^{\circ} \mathrm{C}$ for $15 \mathrm{sec}$ and $60^{\circ} \mathrm{C}$ for $45 \mathrm{sec}$. The RT-qPCR kit and protocol for miR-181a have been described in detail previously (7). RT-qPCR was detected using the ABI PRISM 7500 Sequence Detection system (Applied Biosystems; Thermo Fisher Scientific, Inc.). Each experiment was repeated three times in duplicate. The relative quantification was calculated using the $2^{-\Delta \Delta C t}$ method (10).

Oligonucleotide construction and transfection. All RNA oligonucleotides were synthesized and purchased from Shanghai GenePharma Co., Ltd. (Shanghai, China). Oligonucleotides sequences used were as follows: miR-181a mimic, AACAUUCAACGCUGUCGGUGAGU; miR-181a inhibitor, ACCAUCGACCGUUGAUUGUACC; negative controls (NC), UUCUCCGAACGUGUCACGUTT and ACG UGACACGUUCGGAGAATT; NC inhibitor, CAGUACUUU UGUGUAGUACAA; MTMR3 siRNA (siMTMR3), GGA AGAUAAGGUGAAGUCAdTdT and UGACUUCACCUU AUCUUCCdTdT; scramble mimic, UUCUCCGAACGUGUC ACGUTT and ACGUGACACGUUCGGAGAATT. Cells were transfected with miR-181a mimic (50 nM), miR-181a inhibitor (100 nM), miRNA NC (50 nM), NC inhibitor (100 nM), siMTMR3 (400 nM) or scramble mimic (400 nM) using Lipofectamine $^{\mathrm{TM}}$ RNAiMAX (Invitrogen; Thermo Fisher Scientific, Inc.). Following $5 \mathrm{~h}$ incubation, the medium in each well was replaced by serum-containing medium. Total RNA and protein was extracted $48 \mathrm{~h}$ following transfection.

Western blot analysis. Total protein was extracted using Cell Lysis reagents (Pierce; Thermo Fisher Scientific, Inc.) and quantified by the bicinchoninic acid method (Pierce; Thermo Fisher Scientific, Inc.). Proteins were separated on $10 \%$ SDS-polyacrylamide gel, and then transferred to a polyvinylidene difluoride membrane (GE Healthcare Life Sciences, Chalfont, UK). The membrane was blocked in $5 \%$ non-fat dry milk in TBS and Tween-20 for $1 \mathrm{~h}$ at room temperature and incubated with anti-MTMR3 antibody (dilution, 1:400; Novus Biologicals, LLC, Littleton, CO, USA; cat. no. H00008897-B01P), anti-microtubule associated protein 1 light chain 3 beta (LC3B) antibody (cat. no. L7543; dilution, 1:500; Sigma-Aldrich; Merck Millipore, Darmstadt, Germany) or anti-GAPDH antibody (; cat. no. ab9485; dilution, 1:2,500; Abcam, Cambridge, UK) overnight at $4{ }^{\circ} \mathrm{C}$, followed by incubation with rabbit anti-mouse $\mathrm{IgG}$ and swine anti-rabbit IgG secondary antibodies, both conjugated with horseradish peroxidase (Dako; Agilent Technologies, Inc., Santa Clara, CA, USA; cat. nos. F00037626 and A00048749) for $50 \mathrm{~min}$ at room temperature. The membranes were developed using the
ECL kit (Pierce; Thermo Fisher Scientific, Inc.) and exposed to X-ray film to visualize the images. The GAPDH gene was used as an internal control. The band intensity was analyzed using Gel-Pro Analyzer software (version 4.0; Media Cybernetics, Inc., Rockville, MD, USA).

Proliferation assay. At $24 \mathrm{~h}$ following transfection, cells were trypsinized and seeded into 96 -well plates at a density of $1 \times 10^{4}$ cells/well in growth medium supplemented with $10 \%$ serum. The cell proliferation was determined at different time points $(24,48,72$ and $96 \mathrm{~h})$ using the MTS kit (CellTiter 96 Aqueous One Cell Proliferation Assay; Promega Corporation) following the manufacturer's instructions, and the absorption was read at $490 \mathrm{~nm}$.

Colony formation assay. At $24 \mathrm{~h}$ after transfection, cells were trypsinized and seeded onto 96-well plates at a density of 200 cells/well. The cells were observed every 24 h. Following 7 days of incubation, the colonies were fixed with $4 \%$ paraformaldehyde and then stained with crystal violet for $15 \mathrm{~min}$. The ELISPOT reader (iSPOT system; Autoimmun Diagnostika $\mathrm{GmbH}$, Strassberg, Germany) was used to read the plates. The colony formation rate was acquired through number of colonies/number of plated cells.

Cell cycle assay. Cells were harvested by trypsinization $48 \mathrm{~h}$ after transfection, washed three times with ice-cold PBS, and fixed with $70 \%$ ethanol overnight at $4^{\circ} \mathrm{C}$. The fixed cells were washed in PBS and subjected to propidium iodide (PI)/RNase A staining followed by flow cytometry (BD Biosciences, Franklin Lakes, NJ, USA). The percentage of cells in each phase of the cell cycle was estimated using Modfit LT software, (version, 3.0; BD Biosciences).

Apoptosis assay. Following $24 \mathrm{~h}$ post-transfection, cells were labeled with Annexin V-fluorescein isothiocyanate and PI (Nanjing KeyGen Biotech. Co., Ltd., Nanjing, China), according to the manufacturer's instructions. Cells were analyzed by flow cytometry (BD Biosciences).

Cell migration and invasion assay. Cell migration assay was performed using $8 \mu \mathrm{m}$-pore size 96 -well MIC Transwell plates (EMD Millipore, Billerica, MA, USA). Following transfection, the cells were resuspended in serum-free medium and $1 \times 10^{5}$ cells in $100 \mu 1$ medium were added to the upper chamber while the lower chamber was filled with complete media as a chemoattractant. The cells were incubated for $24 \mathrm{~h}$ at $37^{\circ} \mathrm{C}$, the cells on the upper surface of the membrane were removed by cotton swabs and the cells attached to the lower surface were fixed in ice-cold methanol for $10 \mathrm{~min}$, and stained with $0.5 \%$ crystal violet solution for $10 \mathrm{~min}$. Then the number of migrated cells on the lower surface of the membrane was counted under a microscope in five fields. For the invasion assay, the MIC plates were initially coated with Matrigel (BD Biosciences) diluted in serum free medium and the same procedures as migration assay were performed.

GFP-LC3 analysis. Cells were transfected with a pSELECT-GFP-LC3 expression vector plasmid (InvivoGen, San Diego, CA, USA) using Lipofectamine reagent 
(Invitrogen; Thermo Fisher Scientific, Inc.) following the manufacturer's instructions. In the starvation experiment, $24 \mathrm{~h}$ after co-transfection of miRNAs and GFP-LC3, cells were incubated for $2 \mathrm{~h}$ in Earle's Balanced Salt solution medium. Cells were fixed with $4 \%$ paraformaldehyde in PBS after the treatments. Images were obtained using a fluorescent microscope. Cells with more than five intense GFP-LC3 puncta were considered autophagic, as LC3 is a marker for autophagosomes, whereas those with diffuse cytoplasmic GFP-LC3 staining were considered non-autophagic. The percentage of GFP-LC3-positive cells were counted in >100 cells.

Statistical analysis. Differences between two groups were analyzed by Student's t-test. Data were represented as the mean \pm standard deviation from three independent experiments. Statistical tests were two-tailed. $\mathrm{P}<0.05$ was considered to indicate a statistically significant difference, and was analyzed using SPSS software (version, 17.0; SPSS, Inc., Chicago, IL, USA).

\section{Results}

miR-181a inhibits MTMR3 expression in AGS cells. A previous study demonstrated that miR-181a directly targeted the 3'-UTR of MTMR3 and suppressed the expression of MTMR3 by dual-luciferase assays in HEK-293T cells. In addition, the study validated that artificial overexpression of miR-181a effectively reduced the levels of MTMR3 protein in SGC-7901 cells (7). To further confirm the interaction between miR-181a and MTMR3 in AGS cells, RT-qPCR and western blot analysis were performed, which demonstrated that transfection of miR-181a led to a decrease in MTMR3 mRNA and protein levels compared with the negative control (Fig. 1A and B). However, inhibition of endogenous miR-181a did not increase the levels of MTMR3 mRNA and protein, suggesting that MTMR3 may be suppressed via other factors in addition to miR-181a. These data suggest that MTMR3 is a target of miR-181a.

Effect of overexpressed miR-181a or silenced MTMR3 on proliferation, colony formation, cell cycle, apoptosis, migration and invasion of AGS cells. To explore the role of miR-181a and MTMR3 on the growth of GC cells, an miR-181a mimic or siMTMR3 was transfected into AGS cells. The results of the MTS assay indicated that the proliferation capacity of AGS cells was promoted by upregulation of miR-181a or downregulation of MTMR3, compared with the respective controls (Fig. 2A). Colony formation assay suggested that the number of colonies was significantly higher for cells transfected with the miR-181a mimic and siMTMR3 compared with their respective controls $(\mathrm{P}<0.05$; Fig. $2 \mathrm{~B}$ and $\mathrm{C})$. To assess whether this effect is mediated through perturbation of the cell cycle, cell cycle distribution analysis was performed. There was no difference identified in cell cycle profile among the four groups (miR-NC, miR-181a, scramble and siMTMR3; Fig. 2D). Furthermore, flow cytometry analysis was used to detect the apoptosis of AGS cells transfected with miR-181a mimic or siMTMR3. It demonstrated that the apoptosis rates were decreased in the miR-181a and siMTMR3 groups compared with the $\mathrm{NC}$ and scramble groups, respectively



Figure 1. miR-181a inhibits MTMR3 expression in AGS cells. (A) Reverse transcription-quantitative polymerase chain reaction analysis of MTMR3 mRNA levels in AGS cells treated as indicated. MTMR3 was normalized to $18 \mathrm{~S}$ rRNA. The results represent the means \pm standard deviation for three independent experiments. ${ }^{*} \mathrm{P}<0.05$ vs. miR-NC and miR-NC inhibitor. (B) Western blots demonstrating the protein expression of MTMR3 protein in AGS cells treated as indicated. Data were normalized to GAPDH. The untreated cell group was set as the reference group. miR, microRNA.

$(\mathrm{P}<0.05$; Fig. 2E and F). Finally, results of the Transwell migration assay revealed that artificial overexpression of miR-181a or reduction of MTMR3 significantly increased the migration potential of AGS cells (Fig. 2G). In addition, a significantly higher number of cells in miR-181a and siMTMR3 groups were found to cross the Matrigel compared with the controls in the invasion assay (Fig. $2 \mathrm{H}$ ). These results indicate that MTMR3 knockdown phenocopied the effect of miR-181a overexpression in promoting the development of AGS.

miR-181a and MTMR3 regulate autophagy in AGS cells. Autophagy was determined by following the redistribution of GFP-LC3 from a diffuse to a punctate pattern or by assessing the conversion of endogenous LC3 protein from the cytosolic LC3-I to the autophagosome associated LC3-II by immunoblotting. As presented in Fig. 3A and B, following co-transfection of the GFP-LC3 plasmid and miR-NC/miR-181a mimic, GFP-LC3 was observed predominantly as diffuse green fluorescence in the cytoplasm, indicating low autophagic activities in both groups. This phenomenon was confirmed by immunoblotting (Fig. 3C). Cells were incubated in EBSS medium for $2 \mathrm{~h}$ to induce autophagy. As presented in Fig. $3 \mathrm{~A}$ and $\mathrm{B}$, overexpression of miR-181a significantly blocked starvation-induced GFP-LC3 dot accumulation. In line with this result, starvation-activated lipidation of LC3-II was attenuated in miR-181a mimic transfection group (Fig. 3C). To further demonstrate the effects of endogenous miR-181a inhibition on autophagy, cells were transfected with miR-181a inhibitor or miR-NC inhibitor and autophagy was observed under non-starved or starved conditions. Results indicated that cells treated with the miR-181a inhibitor exhibited a significantly higher percentage of punctate 
A


G

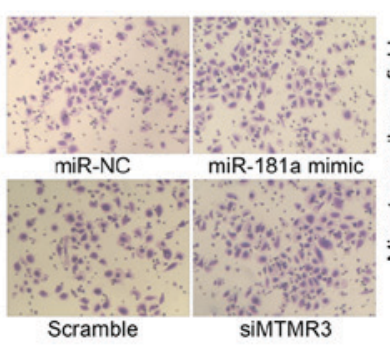

B

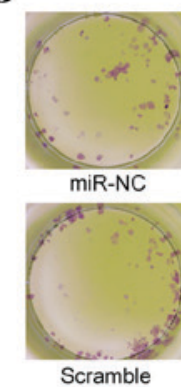

E


Scramble



siMTMR3



miR-181a mimic

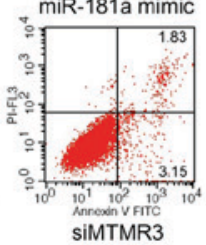

C



F

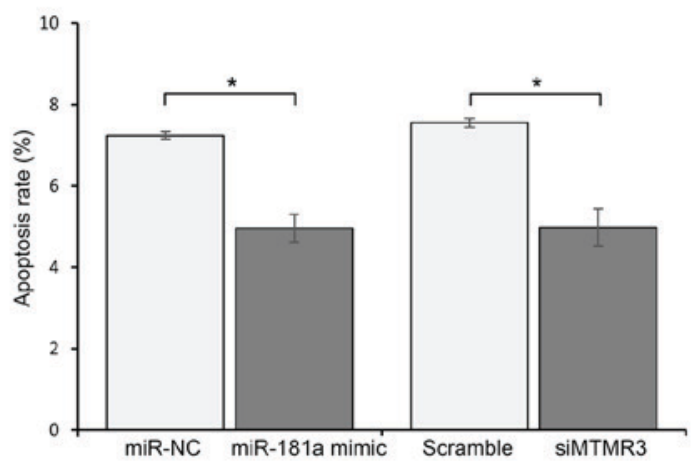

H
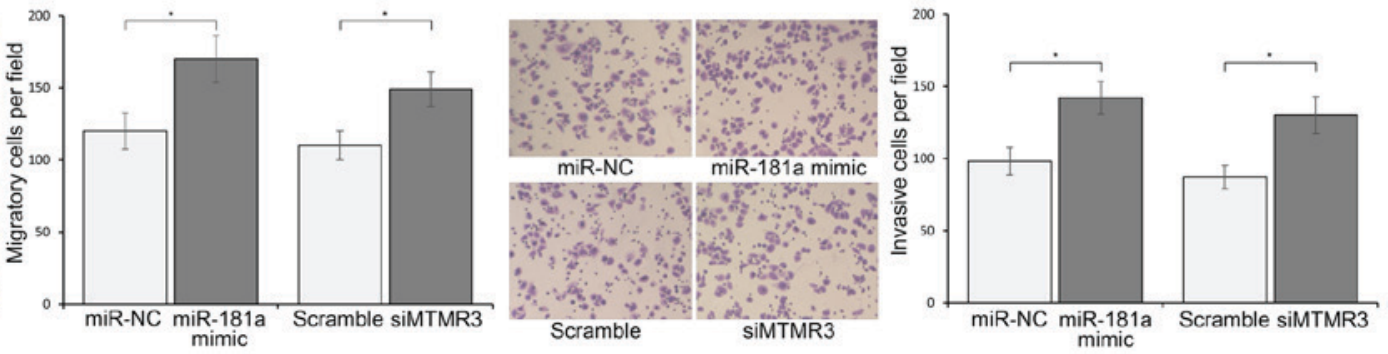

Figure 2. Overexpressed miR-181a or silenced MTMR3 promote proliferation, colony formation, migration and invasion, and inhibit apoptosis of AGS cells. (A) MTS assay showing the proliferation of AGS cells when treated as indicated, measured at 24, 48, 72 and $96 \mathrm{~h}$ following transfection. (B) Colony formation of AGS cells treated as indicated. The colonies were counted on day 7 following transfection. (C) The colony formation rate was acquired through number of colonies/number of planted cells. (D) Cell cycle analysis by flow cytometry. There was no difference in cell cycle distribution among four groups. (E) Flow cytometric assay for the evaluation of apoptosis of AGS cells treated as indicated. Annexin V-FITC $/ \mathrm{PI}^{-}$population (lower right quadrant in diagram) indicated early stage apoptosis, and Annexin V-FITC $/ \mathrm{PI}^{+}$population (upper right quadrant in diagram) indicated late stage apoptosis. (F) Total apoptosis rate was equal to early stage apoptosis + late stage apoptosis. (G) Migration and (H) invasion assay of AGS cells treated as indicated. After $24 \mathrm{~h}$, the number of cells that had migrated/invaded through the membrane were counted under a microscope using five random fields (magnification, x100). The results represent the means \pm standard deviation for three independent experiments. " $\mathrm{P}<0.05$ miR-181a mimic vs. miR-NC, and siMTMR3 vs. Scramble. siMTMR3, myotubularin related protein 3 small interfering RNA; miR, microRNA; NC, negative control; FITC, fluorescein isothiocyanate; PI, propidium iodide.

GFP and LC3-II conversion than cells of miR-NC inhibitor group. In addition, the inhibition of miR-181a further increased autophagic activities during starvation compared with NC (Fig. 3A and B). The role of miR-181a target gene MTMR3 in autophagy was then evaluated. The effect of silenced MTMR3 was similar to that of overexpressed miR-181a, although to a lesser extent (Fig. 3A-C). Knockdown of MTMR3 significantly suppressed starvation-induced autophagy. Together, these findings suggested that miR-181a is a negative regulator of autophagy, partially mediated by inhibition of MTMR3.

\section{Discussion}

Various previous studies have demonstrated that the aberrant expression of miRNAs is implicated in human malignancies $(2,11,12)$. Identification of cancer-specific miRNAs and their targets is critical for understanding tumorigenesis, and may be important for defining novel therapeutic targets. miR-181a, a member of the miR-181 family, is involved in various events, including development, differentiation, hematopoiesis and immune modulation $(13,14)$. The diverse functions of miR-181 in human carcinogenesis may be due to different target genes, depending on the tissue or cellular environment. miR-181a is downregulated in lung cancer (15), leukemia (16) and glioblastoma (17) and acts as a tumor suppressor by targeting KRAS (18), Bcl-2 (19) and PLAG1 (16). In addition, miR-181a is identified as an oncogene by targeting ATM serine/threonine kinase (20), CDX2 (13) and KLF6 (8), and is upregulated in pancreatic cancer (21), hepatocellular carcinoma (13) and gastric cancer (22). The present study revealed that miR-181a functioned as an oncogene in GC cells. Overexpression of miR-181a enhanced the capacity of AGS cells for proliferation, 



C
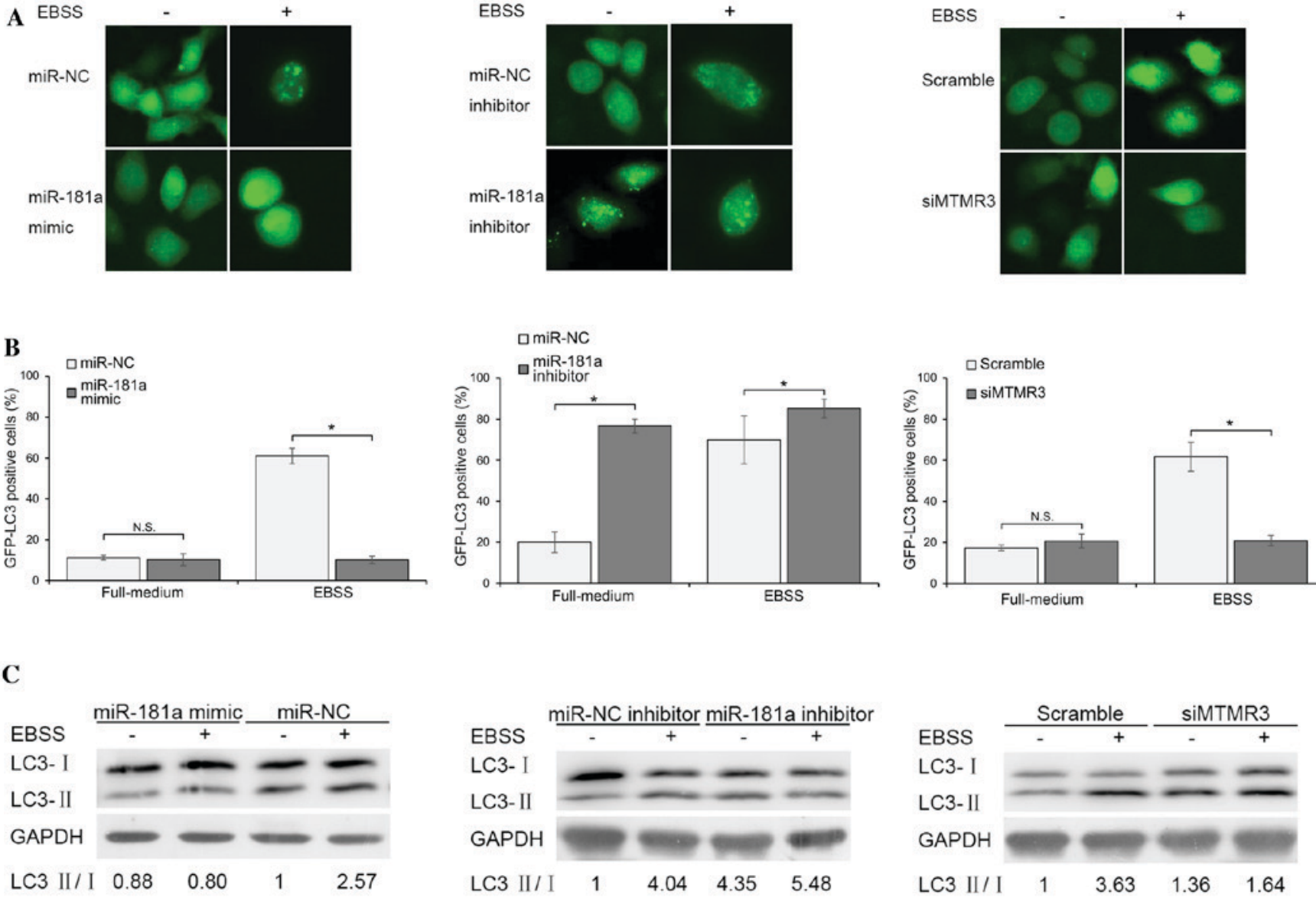

Figure 3. Overexpressed miR-181a or silenced MTMR3 blocks starvation-induced autophagy and inhibition of miR-181a induces autophagy in AGS cells. (A) At $24 \mathrm{~h}$ after cotransfection of miRNAs/siRNA and GFP-LC3 plasmid, autophagic activities were observed under no starvation (full-medium) or starvation (EBSS medium) conditions using fluorescence microscopy (magnification, x200). Cells with more than five intense GFP-LC3 puncta were considered autophagic, whereas those with diffuse cytoplasmic GFP-LC3 staining were considered non-autophagic. (B) Quantitative analysis of the GFP-LC3 positive cells. * P $<0.05$ vs. miR-NC. (C) Western blot results of AGS cells treated as indicated. LC3-II/ LC3-I densitometric ratios were indicated. GAPDH was used as a loading control. EBSS negative miR-NC/scramble group was set as the reference group. EBSS, Earle's Balanced Salt solution medium; miR, microRNA; NC, negative control; siMTMR3, myotubularin related protein 3 small interfering RNA; LC3, microtubule associated protein 1 light chain 3 beta.

colony formation, migration and invasion, whereas it attenuated apoptosis and autophagy. Similarly, Chen et al (22) indicated that enforced expression of miR-181a promoted GC cells proliferation ability. Tekirdag et al (14) suggested that overexpression of miR-181a results in the attenuation of starvation- and rapamycin-induced autophagy in MCF-7, Huh-7 and K562 cells. Furthermore, inhibition of endogenous miR-181a stimulated autophagy. Given that a single miRNA has many different targets, the authors hypothesize that miR-181a targets multiple genes during the regulation of the malignant biological behavior of GC cells, and MTMR3 is one of these targeted genes.

MTMR3 is a member of myotubularin-related protein family, with at least 11 phosphatidylinositol 3-phosphate (PI3P) phosphatases proteins in human. The synthesis of PI3P is required for the initiation of autophagy (23). Autophagy is a cellular degradation pathway for the clearance of damaged or superfluous proteins and organelles. The role of autophagy in cancer is a double-edged sword (24). Certain reports indicate that autophagy serves as a cell survival mechanism, whilst others suggested that autophagy induces autophagic cell death $(25,26)$. A recent study demonstrated that MTMR3 decreased pattern recognition receptor (PRR)-induced PI3P and autophagy levels in monocyte-derived macrophages. In MTMR3-deficient macrophages, reducing the enhanced autophagy or restoring nuclear factor- $x \mathrm{~B}$ signaling rescued PRR-induced cytokines. Thus, modulation of MTMR3 levels may provide a therapeutic benefit in inflammatory bowel disease (27). Another study demonstrated that MTMR3 expression was significantly reduced in colonic biopsies from ulcerative colitis patients (28). Because autophagy may be a tumor suppressor or promoter, the exact function of MTMR3 in cancer remains elusive. A report highlighted that microsatellite instability in MTMR3 may serve a role in the development of gastric and colorectal cancer (29). Another study indicated that MTMR3 was a miR-99a target, and reduced MTMR3 expression in the oral cancer line significantly attenuated cell proliferation, migration, and invasion (26). In addition, miR-100 was demonstrated to increase proliferation and decrease apoptosis of breast cancer cells via targeting MTMR3 (30). The data presented in the current study demonstrated that silencing MTMR3 led to promotion of proliferation, colony formation, migration and invasion, and inhibition of apoptosis and autophagy of GC cells. Further studies are required to clarify the in vivo role of MTMR3 in GC. 
In summary, the current work provides evidence that miR-181a and MTMR3 are important during tumorigenesis and progression of AGS cells. In addition, miR-181a was identified as a novel autophagy regulating miRNA and may act by targeting MTMR3. The results may help understand the potential molecular mechanisms of gastric cancer development and may have potential therapeutic value in the future.

\section{Acknowledgements}

This study was supported by the National Natural Science Foundation of China (grant no. 81302078).

\section{References}

1. Torre LA, Bray F, Siegel RL, Ferlay J, Lortet-Tieulent J and Jemal A: Global cancer statistics, 2012. CA Cancer J Clin 65: 87-108, 2015

2. Wan X, Ding X, Chen S, Song H, Jiang H, Fang Y, Li P and Guo J: The functional sites of miRNAs and lncRNAs in gastric carcinogenesis. Tumour Biol 36: 521-532, 2015.

3. Brodersen P and Voinnet O: Revisiting the principles of microRNA target recognition and mode of action. Nat Rev Mol Cell Biol 10: 141-148, 2009.

4. Shen J, Xiao Z, Wu WK, Wang MH, To KF, Chen Y, Yang W, Li MS, Shin VY, Tong JH, et al: Epigenetic silencing of miR-490-3p reactivates the chromatin remodeler SMARCD1 to promote Helicobacter pylori-induced gastric carcinogenesis Cancer Res 75: 754-765, 2015.

5. Yu Z, Zhang W and Deng F: MicroRNA-577 inhibits gastric cancer growth by targeting E2F transcription factor 3. Oncol Lett 10: 1447-1452, 2015.

6. Song $\mathrm{H}, \mathrm{Xu} \mathrm{W}$, Song J, Liang Y, Fu W, Zhu XC, Li C, Peng JS and Zheng JN: Overexpression of Lin28 inhibits the proliferation, migration and cell cycle progression and induces apoptosis of BGC-823 gastric cancer cells. Oncol Rep 33: 997-1003, 2015.

7. Lin Y, Nie Y, Zhao J, Chen X, Ye M, Li Y, Du Y, Cao J, Shen B and $\mathrm{Li} \mathrm{Y}$ : Genetic polymorphism at miR-181a binding site contributes to gastric cancer susceptibility. Carcinogenesis 33: 2377-2383, 2012.

8. Zhang X, Nie Y, Du Y, Cao J, Shen B and Li Y: MicroRNA-181a promotes gastric cancer by negatively regulating tumor suppressor KLF6. Tumour Biol 33: 1589-1597, 2012.

9. Henderson P, van Limbergen JE, Wilson DC, Satsangi J and Russell RK: Genetics of childhood-onset inflammatory bowel disease. Inflamm Bowel Dis 17: 346-361, 2011.

10. Livak KJ and Schmittgen TD: Analysis of relative gene expression data using real-time quantitative PCR and the 2(-Delta Delta C(T)) Method. Methods 25: 402-408, 2001.

11. Ueda T, Volinia S, Okumura H, Shimizu M, Taccioli C, Rossi S, Alder H, Liu CG, Oue N, Yasui W, et al: Relation between microRNA expression and progression and prognosis of gastric cancer: A microRNA expression analysis. Lancet Oncol 11: $136-146,2010$

12. Li Y, Kuscu C, Banach A, Zhang Q, Pulkoski-Gross A, Kim D, Liu J, Roth E, Li E, Shroyer KR, et al: miR-181a-5p inhibits cancer cell migration and angiogenesis via downregulation of matrix metalloproteinase-14. Cancer Res 75: 2674-2685, 2015.
13. Ji J, Yamashita T, Budhu A, Forgues M, Jia HL, Li C, Deng C, Wauthier E, Reid LM, Ye QH, et al: Identification of microRNA-181 by genome-wide screening as a critical player in EpCAM-positive hepatic cancer stem cells. Hepatology 50: 472-480, 2009.

14. Tekirdag KA, Korkmaz G, Ozturk DG, Agami R and Gozuacik D: MIR181A regulates starvation- and rapamycin-induced autophagy through targeting of ATG5. Autophagy 9: 374-385, 2013.

15. Gao W, Shen H, Liu L, Xu J, Xu J and Shu Y: MiR-21 overexpression in human primary squamous cell lung carcinoma is associated with poor patient prognosis. J Cancer Res Clin Oncol 137: 557-566, 2011.

16. Pallasch CP, Patz M, Park YJ, Hagist S, Eggle D, Claus R, Debey-Pascher S, Schulz A, Frenzel LP, Claasen J, et al: miRNA deregulation by epigenetic silencing disrupts suppression of the oncogene PLAG1 in chronic lymphocytic leukemia. Blood 114: 3255-3264, 2009.

17. Ciafrè SA, Galardi S, Mangiola A, Ferracin M, Liu CG, Sabatino G, Negrini M, Maira G, Croce CM and Farace MG: Extensive modulation of a set of microRNAs in primary glioblastoma. Biochem Biophys Res Commun 334: 1351-1358, 2005.

18. Shin KH, Bae SD, Hong HS, Kim RH, Kang MK and Park NH: miR-181a shows tumor suppressive effect against oral squamous cell carcinoma cells by downregulating K-ras. Biochem Biophys Res Commun 404: 896-902, 2011.

19. Zhu W, Shan X, Wang T, Shu Y and Liu P: miR-181b modulates multidrug resistance by targeting BCL2 in human cancer cell lines. Int J Cancer 127: 2520-2529, 2010.

20. Wang Y, Yu Y, Tsuyada A, Ren X, Wu X, Stubblefield K, Rankin-Gee EK and Wang SE: Transforming growth factor- $\beta$ regulates the sphere-initiating stem cell-like feature in breast cancer through miRNA-181 and ATM. Oncogene 30: 1470-1480, 2011.

21. Zhang P, Guo Z, Hu R, He X, Jiao X and Zhu X: Interaction between microRNA-181a and TNFAIP1 regulates pancreatic cancer proliferation and migration. Tumour Biol, 2015.

22. Chen G, Shen ZL, Wang L, Lv CY, Huang XE and Zhou RP: Hsa-miR-181a-5p expression and effects on cell proliferation in gastric cancer. Asian Pac J Cancer Prev 14: 3871-3875, 2013.

23. Vergne I and Deretic V: The role of PI3P phosphatases in the regulation of autophagy. FEBS Lett 584: 1313-1318, 2010.

24. Mathew R, Karantza-Wadsworth V and White E: Role of autophagy in cancer. Nat Rev Cancer 7: 961-967, 2007.

25. White E: Deconvoluting the context-dependent role for autophagy in cancer. Nat Rev Cancer 12: 401-410, 2012.

26. Kuo YZ, Tai YH, Lo HI, Chen YL, Cheng HC, Fang WY, Lin SH, Yang CL, Tsai ST and Wu LW: MiR-99a exerts anti-metastasis through inhibiting myotubularin-related protein 3 expression in oral cancer. Oral Dis 20: e65-e75, 2014

27. Lahiri A, Hedl M and Abraham C: MTMR3 risk allele enhances innate receptor-induced signaling and cytokines by decreasing autophagy and increasing caspase- 1 activation. Proc Natl Acad Sci USA 112: 10461-10466, 2015.

28. Imielinski M, Baldassano RN, Griffiths A, Russell RK, Annese V, Dubinsky M, Kugathasan S, Bradfield JP, Walters TD, Sleiman $\mathrm{P}$, et al: Common variants at five new loci associated with early-onset inflammatory bowel disease. Nat Genet 41: 1335-1340, 2009.

29. Song SY, Kang MR, Yoo NJ and Lee SH: Mutational analysis of mononucleotide repeats in dual specificity tyrosine phosphatase genes in gastric and colon carcinomas with microsatellite instability. APMIS 118: 389-393, 2010.

30. Gong Y,He T, Yang L, Yang G, Chen Y and Zhang X: The role of miR-100 in regulating apoptosis of breast cancer cells. Sci Rep 5: $11650,2015$. 